\title{
Tuning Local Hydration Enables a Deeper Understanding of Protein-Ligand Binding: The PP1-Src Kinase Case
}

\author{
Andrea Spitaleri, Syeda R. Zia, Patrizio Di Micco, Bissan Al-Lazikani, Miguel A. Soler,* \\ and Walter Rocchia*
}

Cite This: J. Phys. Chem. Lett. 2021, 12, 49-58

Read Online

ABSTRACT: Water plays a key role in biomolecular recognition and binding. Despite the development of several computational and experimental approaches, it is still challenging to comprehensively characterize water-mediated effects on the binding process. Here, we investigate how water affects the binding of Src kinase to one of its inhibitors, PP1. Src kinase is a target for treating several diseases, including cancer. We use biased molecular dynamics simulations, where the hydration of predetermined regions is tuned at will. This computational technique efficiently accelerates the SRC-PP1 binding simulation and allows us to identify several key and yet unexplored aspects of the solvent's role. This study provides a further perspective on the binding phenomenon, which may advance the current drug design approaches for the development of new kinase inhibitors.

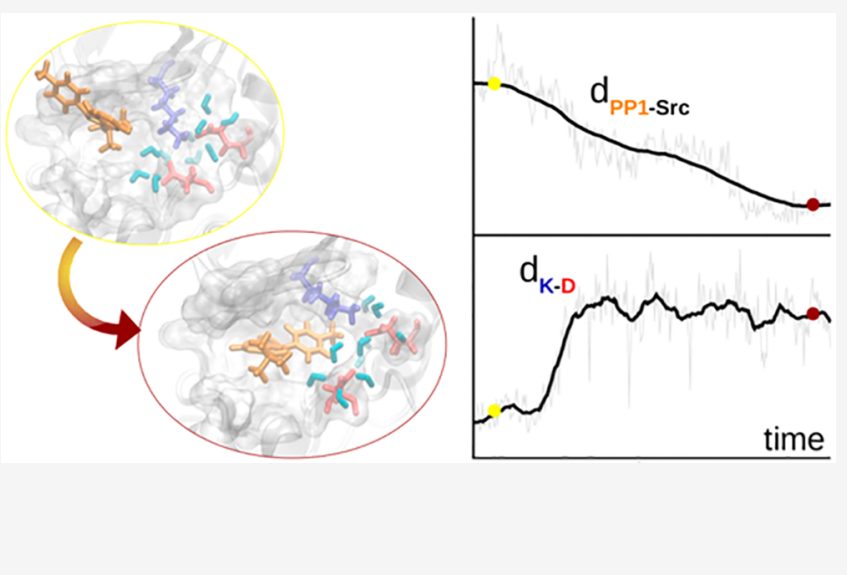

$\mathrm{M}$ olecular recognition is the basis of every biological process. Biomolecules evolved in an aqueous environment, so they largely exploit the properties of water to achieve the binding affinity and specificity required for an efficient functioning. Three important phenomena arising from the properties of water are dielectric screening, hydrophobic interaction, and viscous drag, which affects diffusivity. Dielectric screening is mostly a product of molecular polarizability and is at the basis of the desolvation penalty. ${ }^{2}$ The desolvation penalty is the energy that binding partners have to yield in order to bury polar and H-bond-forming groups, therefore depriving them of direct interactions with water molecules. ${ }^{3}$ Hydrophobic interactions in biomolecular systems are water-mediated forces that come into play when hydrophobic groups on the solute's surface are present. They are more difficult to characterize and are not always intuitive., Hydrophobic interactions are thought to play a primary role in solving Levinthal's paradox of protein folding, and they are crucial to biomolecular recognition. ${ }^{1,6-8}$ It is important to understand the mechanistic details of water's role in molecular recognition. This is true for fundamental science (i.e., Physical Chemistry and Biology) but also for applied drug design, where this understanding can be used to engineer new molecules with desired binding profiles. For these reasons, this field has been continuously a subject of active research. ${ }^{9-13}$

It is challenging to experimentally characterize the properties of water at the molecular level. ${ }^{14}$ The available structures of biomolecules typically reveal multiple water sites near the biomolecular surface, buried inside cavities, or at binding

interfaces. Water sites can be detected in the crystal state by Xray (with a resolution of at least $2.0 \AA)^{15}$ and neutron diffraction or in solution by nuclear magnetic resonance (NMR) and other spectroscopy approaches. ${ }^{16}$ NMR spectroscopy allows probing proteins in aqueous solution at room temperature, and it can also be exploited to study watermediated protein-ligand interactions. ${ }^{17}$ Infrared spectroscopy is another powerful tool for water characterization. ${ }^{18}$ Specifically, terahertz absorption spectroscopy can probe the different fluctuating orientations of molecular dipoles and the collective intermolecular vibrations of the hydrogen-bond network in bulk water and in water molecules interacting with solutes. ${ }^{19}$

Theoretical and computational approaches can more easily trace the individual behavior of solvent molecules, including water molecule orientation, tumbling, and residence time, but the inherent complexity of the recognition process makes understanding their role in this context a significant challenge. $^{13,20-22}$ While an exhaustive examination of the related literature, which is massive, goes beyond the scope of this work, we would like to distinguish reductionist approaches

Received: October 8, 2020

Accepted: December 3, 2020

Published: December 10, 2020 
(a)

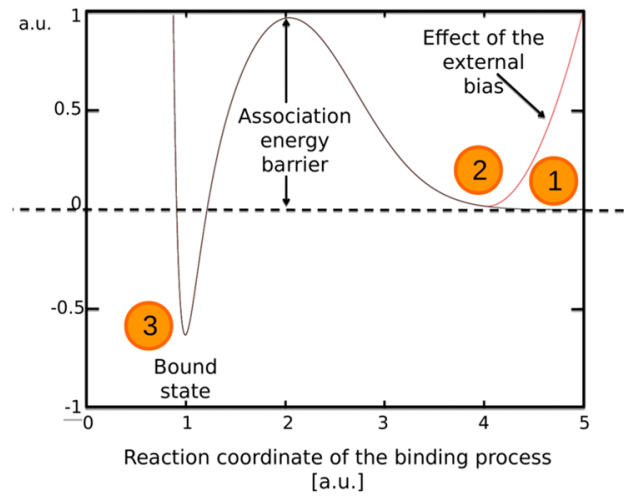

(c)

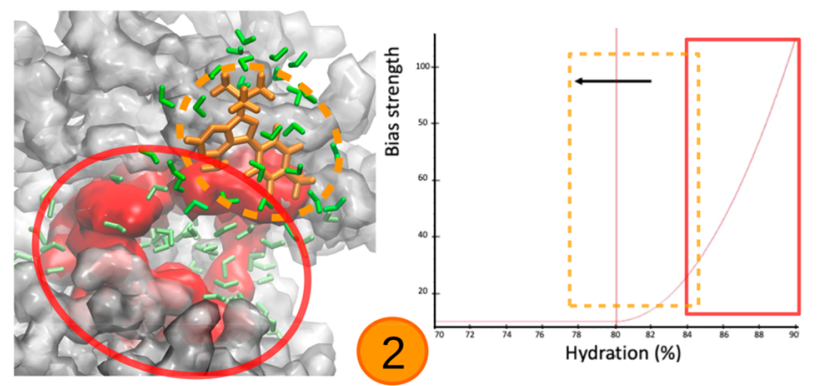

(b)
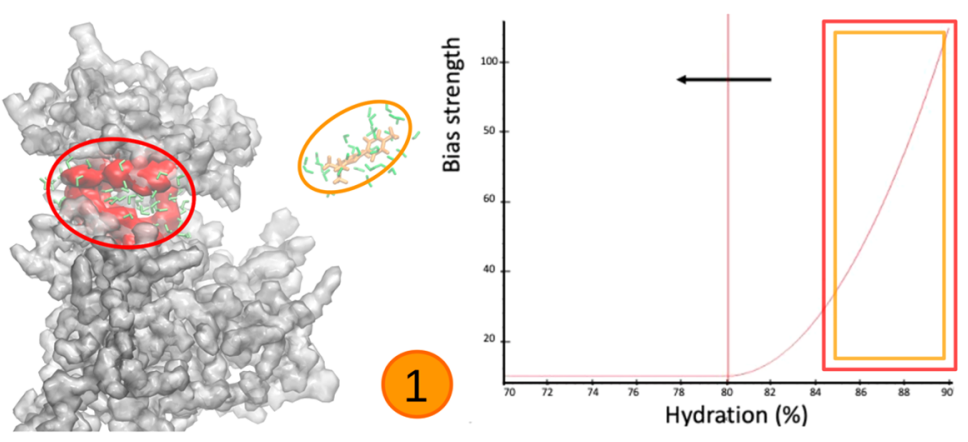

(d)

Figure 1. Schematic representation of the free-energy profile (a) and the dehydration bias mechanism (b-d) during the binding process. At the initial stage, 1 , when the ligand is in the bulk, a semiparabolic bias is applied over the values of the electrostatic collective variables of the ligand and the Src binding site (BS). The combined water repulsion of these regions increases the effective concentration of the ligand in front of the site, 2, and, therefore, the likelihood of binding. The vertex of the bias is gradually shifted in the direction of the black arrow from $100 \%$ of the unbound value to the desired one. The external force automatically switches off as soon as the target hydration level is reached, 3. Localized dehydration can also affect the height of the association energy barrier in both directions, depending on the properties of the specific binding system.

from atomistic-level approaches. The former attempt to derive general principles from studies performed on simplified and/or schematized systems (e.g., host-guest pairs), using implicit solvent descriptions or concepts typical of mesoscale physics $(\geq \mu \mathrm{m})$ such as contact angles and liquid/vapor phases. ${ }^{23-25}$

The latter describe biomolecular systems with the greatest available level of detail, which is normally the atomistic structure, and break down the full binding process into a collection of punctual events, such as the formation and breaking of $\mathrm{H}$ bonds (see, e.g., Balius et al. ${ }^{26}$ and refs therein). A complete understanding of the recognition and binding mechanisms would require going from the big picture to the finer details, which can be a daunting task.

One of the most fascinating results of reductionist studies is that biological systems tend to function at the border of phase transitions in order to promptly react to minor environmental changes. $^{27}$ With regard to hydrophobic interactions, the density of water near partially hydrophobic binding interfaces appears to be in a highly fluctuating state similar to that of liquid-vapor interfaces. This makes the desolvation propensity sensitive to subtle changes in surface conformation, topology, and chemistry, which significantly impacts biomolecular function. $^{24,28,29}$ The energetic characterization of biomolecular association must therefore include a thorough evaluation of solvent-related contributions, the behavior of which can be counterintuitive and can exhibit a high degree of entropyenthalpy compensation. ${ }^{25,30-32}$ Molecular dynamics (MD) is the appropriate computational tool for dynamically characterizing any binding process at the atomistic level. ${ }^{273-35}$ The unique advantage of detailed atomistic simulations is that they can identify specific molecular determinants of binding, including solvent-related ones, and offer useful clues about how to chemically modify a candidate drug. ${ }^{22,29,36-39}$ Ideally, one would exhaustively sample the conformational space of a given process to achieve a thorough characterization. However, this use of MD creates some challenges. Recognition and binding are so-called "rare" or "activated" events, for which extensive sampling is often computationally unaffordable. Nevertheless, plain MD simulations have achieved this in several cases. ${ }^{39-43}$ In these instances, observations based on a number of long simulations, lasting from a handful to hundreds of microseconds $(\mu \mathrm{s})$, provided many useful insights, although they were statistically insufficient in terms of inferring solid conclusions about the full binding mechanism. A further difficulty of computational studies is that static crystal structures, albeit sometimes multiple conformations are available, are the only option for benchmarking their results. Despite these limitations, computational simulations have generated fruitful discussions about the impact of solvent perturbation on the binding affinity and the kinetics of congeneric ligands binding to the same target. ${ }^{20,44}$

The mechanism of drugs binding to protein kinases is an ideal example for demonstrating the active role of water molecules. ${ }^{45,46}$ Protein-Ligand binding in protein kinases can be described as a two-step model representative of a general induced-fit mechanism. ${ }^{47}$ The ligand must first reach the proximity of the entrance to the cavity containing the binding site, then enter through a better-defined path to complete the process (see Figure 1a)..$^{39,48}$ Protein kinases are enzymes involved in cellular signaling. They are attractive targets for treating several diseases, including cancer, cardiovascular diseases, central nervous system disorders, diabetes, and 
inflammatory diseases. ${ }^{49-51}$ Because of the existence in the literature of experimental and computational knowledge, we chose to study Src kinase interacting with its PP1 inhibitor. This system is deemed to be representative of a large class of inhibition mechanisms in the kinase family. Abnormally increased activity of c-Src kinase is associated with various forms of cancer in humans, including cancers of the lungs, liver, colon, and pancreas. ${ }^{52}$

Our method tries to find an intermediate position between reductionist and atomistic approaches. We use our previously developed hydration collective variable ${ }^{53}$ to induce, within the simulative framework of explicit solvent MD, a controlled repulsion between predetermined regions of the solute and the water molecules in their surroundings. By tuning the effective hydrophobicity of the PP1 ligand and of the Src kinase cognate binding site (see Figure $1 \mathrm{~b}-\mathrm{d}$ and Figure S1), we increase the likelihood of observing binding events during the simulation and we analyze the mechanistic impact of hydration on specific determinants of binding. We discuss the biophysical and biological implications of our findings.

We performed 64 plain and 198 biased MD simulations, each $\sim 35 \mathrm{~ns}$ long, amounting to a total of $\sim 9.2 \mu \mathrm{s}$ of simulations of the Src-PP1 biomolecular system starting from the unbound state. As detailed in the Methods section in the Supporting Information, the bias involves applying a repulsive force between predetermined regions of the system (here, the heavy atoms of the ligand and the binding site, see Figure S1) and the surrounding water molecules. The effect of this force on the water region nearby the entrance of the site was first analyzed in the trajectory where the ligand never got close to the binding site. The analysis considered the water behavior at distances from the center of the binding site ranging from zero to slightly more than $1 \mathrm{~nm}$. Here, as expected, the bias slightly lowers the density of water (see Figure S2) and causes an increase of the fluctuations of water density, especially near the entrance of the site (Figure S3), similarly to what occurs around hydrophobic sites. ${ }^{54}$ The method is designed in a way that, when the water density around any biased region goes below the corresponding predefined threshold, the bias automatically switches off. This is what happens when the ligand reaches the binding site and both move to a lesshydrated state. The rationale for this is to allow for the recovery of an unbiased evolution after the association energetic barrier has been overcome (see Figure 1). The choice of the hydration thresholds is somehow heuristic and benefits from the experience achieved during the application of the method developed in ref 53, where the residual hydration of the ligand and the BS upon binding were observed. They are expressed as percentages, namely, $50 \%$ for the ligand and $80 \%$ for the BS, of the unperturbed hydrations. Our analysis focuses on three aspects: (i) the likelihood of observing a successful binding event, (ii) the mechanistic molecular details of the recognition and binding of PP1 to Src, and (iii) whether these observations can be generalized to the kinase protein superfamily.

\section{MODULATING HYDRATION INCREASES BINDING OCCURRENCE IN THE SIMULATION}

Figure 2 shows the distribution of the root-mean-square deviation (RMSD) values averaged over the last $1 \mathrm{~ns}$ of the simulation for each replica with respect to the PP1-Src native complex (distances between the center of masses of PP1 and the Src binding site, PP1-SrcBS, are shown in Figure S4; see

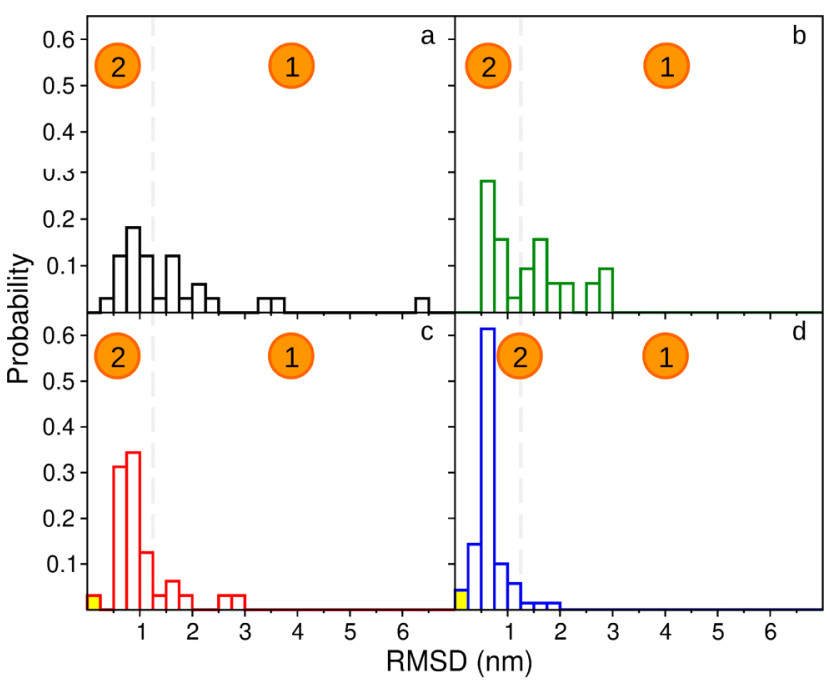

Figure 2. Distribution of the RMSD values for each set of (a) unbiased, (b) single bias, (c) double bias, and (d) double-delayed bias trajectories. Orange circles indicate the ligand when unbound, 1 , and while approaching the binding site, 2 . The probability of native binding events (case 3) is highlighted in yellow for all sets of trajectories.

Supporting Information for a detailed explanation of their calculation). As expected, the inhibitor did not reach the Src binding site in any of the short unbiased trajectories (see Figure 2a). In the single bias, where the dehydration bias is applied to PP1 only, the ligand gets close to the SrcBS entrance in most trajectories (case 2, see Figure 1c), but it is never observed to complete the binding process. The application of this protocol narrowed the distribution of PP1-SrcBS distances and RMSD of the unbiased trajectories to lower values (see Figures $2 \mathrm{~b}$ and $\mathrm{S} 4 \mathrm{~b}$ ). In the single-bias trajectories on average the ligand always remains attached to the protein (see Figure S5). The completion of the binding (from case 2 to 3, see Figure 1) is facilitated in the double-bias protocol, where we also apply a dehydration bias to the SrcBS. This allowed one trajectory to achieve the native binding conformation (reaching a value of $0.67 \AA$ for the RMSD of the ligand with respect to (wrt) crystal averaged along the last ns) and increased the number of trajectories in which PP1 ends up in case 2, that is, near the SrcBS (see Figures $2 \mathrm{c}$ and S4c). The same trend was observed in the double-delayed protocol, that is, with a delayed bias over the SrcBS, where three native binding events were achieved (last ns average RMSD wrt crystal of 0.95, 0.90, and $0.87 \AA$, respectively, see Figures $2 \mathrm{~d}$ and $\mathrm{S} 4 \mathrm{~d}$ ). However, the analysis of the trajectories with the double-delayed bias indicates the presence of several false positives, that is, poses characterized by short ligand-SrcBS distance but large RMSD values (see Figures $2 \mathrm{~d}$ and S4d). The double-delayed bias results in an overintense bias that provoked the shift of the water molecules that formed $\mathrm{H}$ bonds with polar and charged residues at the SrcBS entrance (Gly344 and Ser345 on one side and the residues Lys295, Glu310, and Asp404 on the other) when PP1 approached this region, hence creating alternative, high-energy entrance pathways for the drug.

\section{BINDING-SITE DEHYDRATION FACILITATES THE COMPLETION OF THE BINDING PROCESS}

The results of the previous section suggest that hydration's role in the last stage of the binding process could be subtler than 

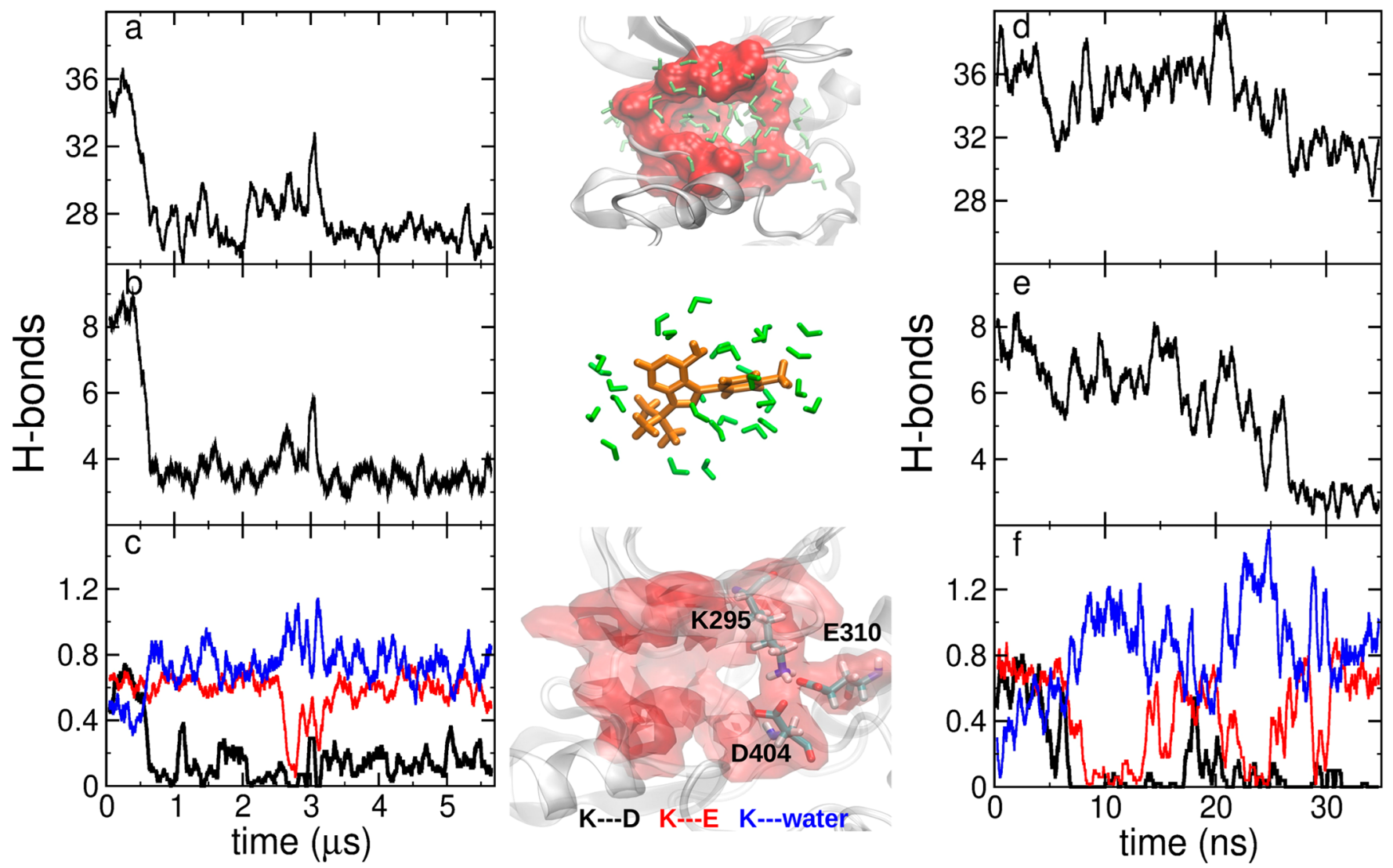

Figure 3. The evolution of the hydration of the targeted regions is evaluated during Simulation 3 from D. E. Shaw Research (a-c) and during the successful double-biased MD trajectory $(\mathrm{d}-\mathrm{f})$. The application of the hydration bias accelerates the entry of the ligand by almost 2 orders of magnitude. The behavior of the hydration in Src-BS (a,d) and PP1 (b, e), estimated by averaging the number of H-bonds with water along time, correlates with that observed for the Lys295-Asp404 (KD) and Lys295-Glu310 (KE) salt bridges, as they must break (especially the KD salt bridge) to allow the entry of PP1 (c, f). For clarity of representation, raw values have been smoothed by averaging over $200 \mathrm{~ns}$ (a-c) and over 0.5 ns $(\mathrm{d}-\mathrm{f})$. As it was done for the H-bonds, the distance used to evaluate the frequencies of formation of salt bridges or of interaction between the $\mathrm{NZ}$ atom of a lysine and a water molecule is of $4 \AA$.

expected. To confirm this, we deepened the analysis of the hydration shell of PP1 and of the Src binding site during the transition from the entrance region and up to the complete binding. We used two plain MD trajectories of PP1 binding to Src kinase, obtained from the work of Shan et al. ${ }^{39}$ Figure $3 \mathrm{a}, \mathrm{b}$ shows the number of $\mathrm{H}$ bonds between the SrcBS and water and between PP1 and water, respectively, for one trajectory, named Simulation 3, as in ref 39. The complete binding, which occurs after $0.6 \mu \mathrm{s}$, is concurrent with a significant decrease in the number of $\mathrm{H}$ bonds formed between water and, respectively, PP1 and the SrcBS. After binding, there is an increased fluctuation in the number of $\mathrm{H}$-bonds, especially for the SrcBS, until a more stable behavior is achieved at $\sim 3 \mu \mathrm{s}$. Similar behavior can be observed in the other simulation by Shan et al., namely, Simulation 1 (see Figure S6a,b). As detailed below, these fluctuations correspond to the dehydration of the binding site. Notably, we observed the same behavior in our successful, that is, leading to a near-native complex, double-biased trajectory (see Figures 3d,e and S7S9a,b). Indeed, our biased trajectories and the unbiased ones from Shan et al. were in good agreement in terms of number of $\mathrm{H}$ bonds between water and PP1 and between water and the SrcBS. This confirms that the applied dehydration bias does not significantly perturb the H-bond interactions between water and the ligand or the SrcBS, while favoring the permanence of the ligand near the protein and increasing the frequency of water entering and abandoning the SrcBS cavity. A further observation on the SrcBS hydration showed that several water molecules did continue to directly interact with the polar residues at the bottom of the SrcBS during PP1 entry (see snapshots in Figure S10 for greater clarity). Analysis of the residence time of these molecules in the cavity during the unbiased Shan et al. binding trajectories provided upper-bound estimates on the order of tens of nanoseconds. Thus, once PP1 enters the cavity, it takes several nanoseconds to clear all the water molecules and achieve the final binding conformation, as it has been noted elsewhere. ${ }^{39,48}$ This kind of mechanism may respond to different functions. On one side, the water molecules could act as a "shield" during binding, attenuating possible repulsive or energetically unfavorable interactions between the drug and the polar side-chain residues. On the other side, the water molecules could help the selectivity toward the correct binding conformation between PP1 and SrcBS, since their presence modifies the accessible conformational space of the cavity. This second explanation is consistent with the results obtained by applying the double-delayed bias, where an excessive or too rapid dehydration bias led to nonnative bindings due to incorrect PP1 orientations inside the cavity (see Figures $2 \mathrm{~d}$ and S4d). 

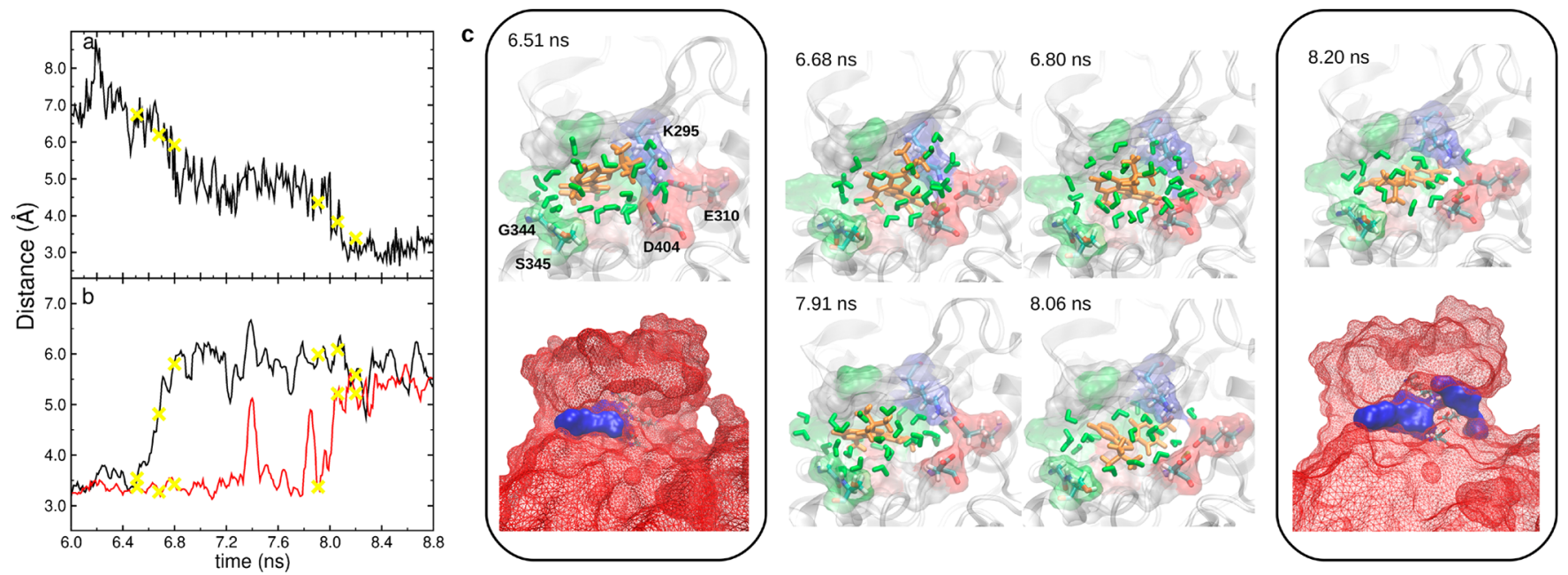

Figure 4. Analysis of the entry of PP1 into the BS of Src kinase. Distance between (a) PP1 and Src BS and (b) Lys295-Asp404 (black) and Lys295Glu310 (red) along the interval in which PP1 achieves the binding site is evaluated in the successful double-biased MD trajectory. Some representative snapshots, represented by yellow crosses in panels (a) and (b), are shown in panel (c) and depict the mechanism of PP1-Src binding. PP1 and its hydration shell as well as the polar (green) and charged (red for negative charge, blue for positive charge) residues of the Src binding site are represented using the Licorice Representation in the molecular visualization software VMD. A molecular surface representation is shown of the cavity size when the salt bridges are formed at $6.51 \mathrm{~ns}$ and broken at $8.20 \mathrm{~ns}$. The pocket and protein surfaces were calculated with Nanoshaper. ${ }^{60}$

\section{A LYS-ASP SALT BRIDGE EMERGES AS A MODULATOR FOR BINDING}

Observing the binding mechanism during the transition between cases 2 and 3 (see Figure 1) usefully elucidates the role of water molecules. In the Shan et al. trajectories and in our biased simulations, the binding of PP1 occurred through the entrance delimited by the hydrophobic patch Leu273 and Tyr340 and the groups Gly344 and Ser345, on one side, and the charged residues Lys295, Glu310, and Asp404, which form the salt bridge Lys295-Glu310 (in short: KE) and the salt bridge Lys295-Asp404 (in short: KD) (see Figure 3). Lys295, located in the beta- 3 secondary structure, is conserved in the protein tyrosine kinase family (Pkinase_Tyr (PF07714)) and forms the salt bridge with Glu310 of the C-helix (also highly conserved in the family Pkinase_Tyr). ${ }^{55}$ In turn, Asp404 is found in the highly conserved Asp-Phe-Gly (DFG) motif located at the beginning of the activation loop. In the active state, its side chain interacts with $\mathrm{Mg}^{2+}$ during adenosine triphosphate (ATP) catalysis. The salt bridge KE is considered primordial and key for the correct functionality of the active state of protein kinases. ${ }^{55-58}$ Computational studies have linked the $\mathrm{KD}$ salt bridge to the flipping transition from the "DFG in" to "DFG out" conformation of the A loop, as a stabilizer of the DFG in conformation. ${ }^{59}$ We followed the behaviors of $\mathrm{KE}$ and $\mathrm{KD}$ along the Shan et al. unbiased binding trajectories (see Figures $3 \mathrm{c}$ and S5c) and during our successful biased ones (see Figures $3 \mathrm{f}$ and $\mathrm{S} 6 \mathrm{c}-\mathrm{S} 8 \mathrm{c}$ ) by comparing Lys295-water interactions with the occurrence of these salt bridges. We found a strong correlation between PP1 entering the cavity and the rupture of the KD salt bridge. As PP1 enters the cavity and begins its dehydration (e.g., see Figure 3b), the hydration of Lys295 and its distance from Asp404 increase (see Figure 3c).

In contrast, the KE salt bridge seems to be less affected by PP1 entering the cavity, although PP1's interaction with the SrcBS leads to greater fluctuations in the distance between the two forming residues (see Figure 3c,f). Moreover, before PP1 enters the cavity, both $\mathrm{KD}$ and $\mathrm{KE}$ salt bridges are quite stable, with a relative frequency of formation of $72 \%$ for $\mathrm{KD}$ and even larger for KE. During association, the $\mathrm{KD}$ bridge becomes mostly disrupted, and its breakage concurs with a significant increase in the SrcBS size (see Figure 4). After the binding, a formed $\mathrm{KD}$ bridge can be observed in $14 \%$ of the remaining simulation.

\section{HYDRATION INFLUENCES THE STABILITY OF THE LYS295-ASP404 BRIDGE AND THE BINDING}

To corroborate the previous observations, we used the successful double-biased trajectories, which have a picosecond temporal resolution, to analyze in more detail the molecular interactions taking place from the time of the ligand's entry until it achieves the native-like pose. In Figures 4 and S10, we characterize the path interval in which PP1 reorients and enters the cavity. We do this by plotting the distance between PP1 and the Src binding site (see Figure 4a) and the distances between the atoms $\mathrm{N}$ of the $\varepsilon$-amino group and $\mathrm{C}$ from the $\beta$ and $\gamma$-carboxyl groups involved in the $\mathrm{KD}$ and $\mathrm{KE}$ salt bridges, respectively (see Figure $4 \mathrm{~b}$ ). Again, these results demonstrate the strong relationship between PP1 entering the cavity and the salt bridge breaking. Along this path, water molecules accompany PP1 while it enters, interacting with different polar and charged groups surrounding the cavity entrance, that is, Gly344 and Ser345 on one side and Lys295, GluE310, and Asp404 on the other (see snapshots in Figures 4c and S9). More importantly, the water molecules surrounding PP1 seem to be responsible for the breaking of the $\mathrm{KD}$ salt bridge (see snapshots at 6.80 and $7.91 \mathrm{~ns}$ in Figure 4c) by interacting with the charged groups. Although we cannot rule out that the rupture of a water network might be (co)responsible for $\mathrm{KD}$ breakage, we observed in our simulations that the high flexibility of the involved residues and their ability for multiple interaction modes hinder the formation of any stable water network. The subsequent increase in distance between both residues leads to the widening of the cavity (as shown in Figure 4c). We found a similar behavior in the two unbiased 

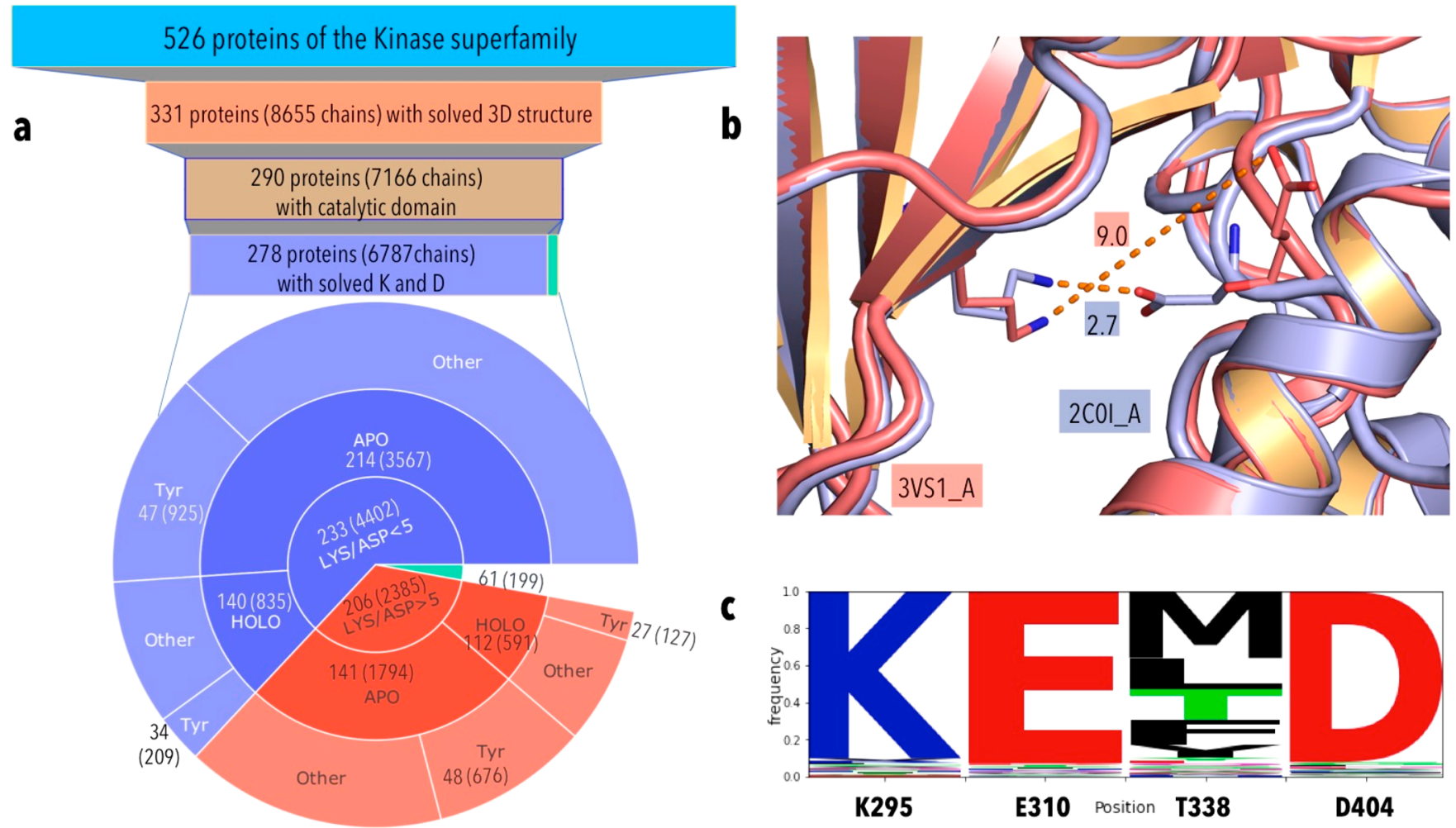

Figure 5. Bioinformatic and structural analysis of the conservation of the $\mathrm{KD}$ bridge within the kinase superfamily. (a) Schematic representation of the down selection process performed on the initial kinase superfamily (upper diagram) and sunburst chart of structural analysis (lower diagram). In blue, structures where the K-D salt bridge is conserved (Lys295 - Asp404 distance > $5 \AA$ ). (b) Structural differences between active (A-loop in red) and inactive (A-loop in blue) conformation of ABL kinase, used as a representative system. Distance, in angstroms, between Lys and Asp residues in the two conformations. (c) Sequence logo of Src kinase key residues.
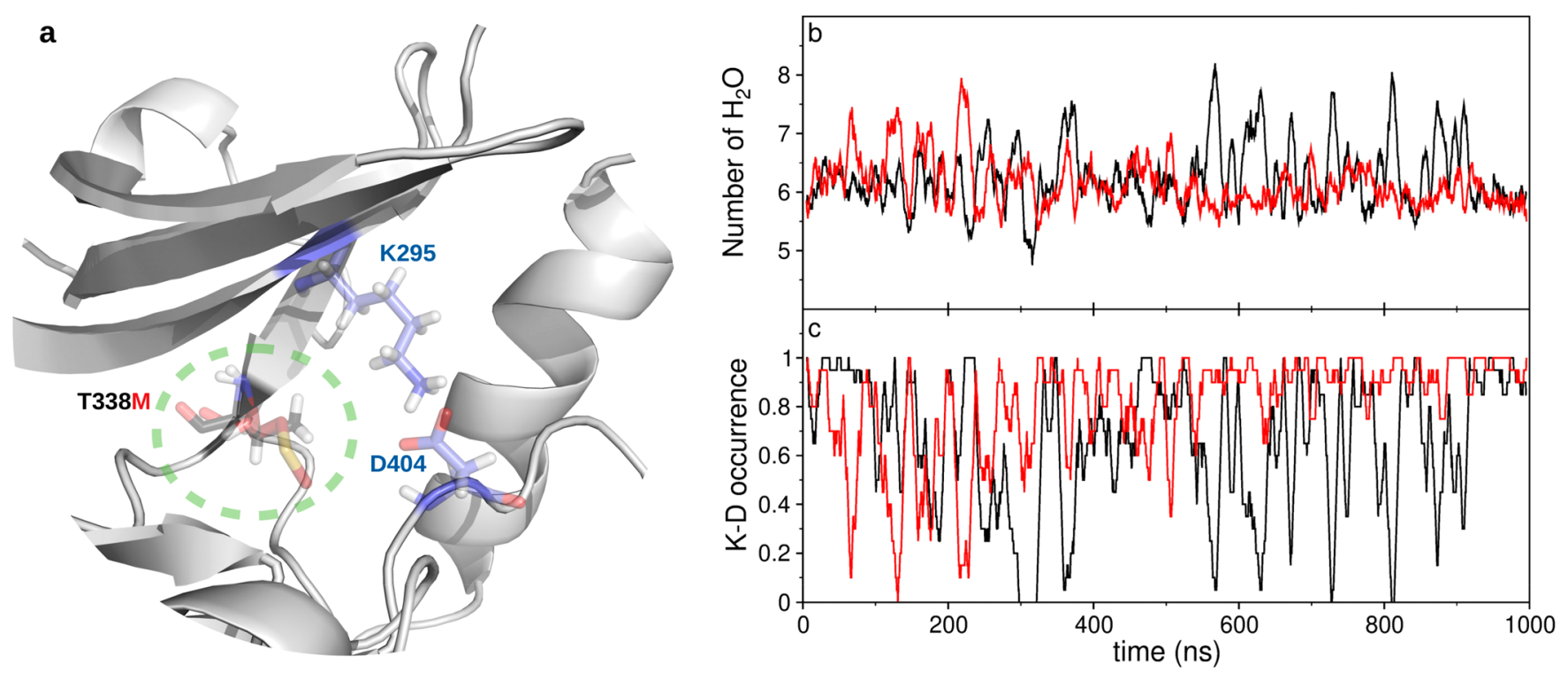

Figure 6. (a) Structural representation of the gatekeeper residue Thr338 overlapped with the mutation Met, and the Lys295-Asp404 salt bridge in the Src binding site. (b) Number of water molecules within $3 \AA$ of the $\mathrm{N}$ of a Lys side chain or any of two O atoms of Asp side chain in the KD salt bridge along the MD trajectories of SRC (black) and SRC Thr338Met (red). (c) Occurrence of the KD salt bridge for the MD trajectories of SRC (black) and SRC Thr338Met (red). Curves in (b, c) were smoothed by averaging over a window of 20 points ( $\pm 5 \mathrm{~ns})$.

trajectories from D. E. Shaw Research (see Figures S11 and S12), although their time resolution of $1 \mathrm{~ns}$ makes difficult a thorough analysis of the entrance event, which occurs in a short time range of 2-3 ns. To support the conjecture that hydration impacts $\mathrm{KD}$ stability, we performed an additional set of biased simulations, in which the $\mathrm{KD}$ salt bridge is dehydrated by up to $50 \%$ of the average value obtained in the absence of PP1.

During the first stages of the simulation, $\sim 40 \%$ of the trajectories started with a broken salt bridge, which is 
compatible with the situations previously observed in the unbiased simulations. However, at $10 \mathrm{~ns}$, when the dehydration bias on the salt bridge achieved the maximum value of $50 \%$, the salt bridge was strongly formed in $\sim 90 \%$ of the trajectories. Afterward, we did not observe any breaking of this salt bridge in our simulations. This provides quantitative support to the intuitive idea that hydration increases the breaking frequency of the salt bridge. We did not observe, in our simulations, any persistent water network around the $\mathrm{KD}$ bridge.

\section{CONSERVATION OF THE KD BRIDGE IN THE KINASES}

The Lys295-Asp404 breakage mechanism upon inhibitor binding may be common to other members of the kinase family due to the high conservation of the residues composing the $\mathrm{KD}$ bridge. To confirm this hypothesis, a large-scale structural analysis has been performed across all the available three-dimensional (3D) structures of kinases superfamily available from the Protein Data Bank (PDB) (22/05/2020). A KD bridge was identified when the equivalent Lys295 (conserved Lys) and Asp404 (DFG Asp) residues are within 5 $\AA$ of each other. The same figure has been used to classify kinase conformations as DFG-in active. ${ }^{61}$

As represented in Figure 5, our analysis showed that equivalent Lys295 and Asp404 residues were found in the 78\% of the available structures (6787 individual chains). A KD bridge was found in 64\% (4402 chains) of these, and the relative structure is classified as DFG-in. To investigate the impact of a ligand on the $\mathrm{KD}$ bridge, we discriminated then between DFG-in kinases with a small molecule in the primary pocket (HOLO) and DFG-in kinases with empty pockets (APO). 3567 DFG-in HOLO chain structures (81\%) were found to show a Lys295 and Asp404 distance compatible with a salt bridge after the binding of a small molecule.

The same analysis was performed taking into account the 1934 structures belonging to the tyrosine protein kinase family only. $47 \%$ (925 chains) was found to preserve the KD bridge when a small molecule is bound on the primary cavity (see Figure 5). These observations support the idea that the $\mathrm{KD}$ bridge can contribute to the association energetic barrier of ligands in a large number of cases.

A further way to explore the biological relevance of our finding is to consider how an important mutation in the binding site of tyrosine kinases (that of the gatekeeper T338M, which brings resistance to some drugs, ${ }^{62}$ affects the stability of the $\mathrm{KD}$ salt bridge). In the $\mathrm{T} 338 \mathrm{M}$ isoform, a hydrophilic residue (Thr) is replaced by a more hydrophobic one (Met) in a mostly hydrophobic cavity (see Figure 6a). This induces a decrement both of the average number of water molecules inside the cavity and also around the $\mathrm{KD}$ salt bridge (see Figure 6b). As observed before (see Figure 3), the occurrence of the KD salt bridge strongly correlates with the decrement in the number of water molecules (or number of water $\mathrm{H}$ bonds) around the side chains of Lys295 and Asp404 (see Figure 6c). In the T338M Src mutant, after a period of equilibration of $\sim 300$ ns upon mutation, the $\mathrm{KD}$ salt bridge remains stable as well as the number of water molecules around the group. In the wild-type Src, each peak in the number of water molecules matches the breaking of the KD salt bridge. This is important in terms of the conformational stability of kinase binding sites, since previous simulations of Shan et al. ${ }^{63}$ have demonstrated that the breaking of the KD salt bridge shifts the DFG loop conformational equilibrium, accelerating its flipping process toward the DFG out inactive form. Moreover, this information is relevant to the work of Hari et al., ${ }^{64}$ who demonstrated experimentally that the nature of the gatekeeper residue regulates the DFG-in and -out conformation equilibrium of the kinases. Therefore, the ability of the hydrophobic gatekeeper residue to stabilize the $\mathrm{KD}$ salt bridge and hence the DFG-in conformational state is an important factor to consider in terms of drug resistance ${ }^{65}$ or when characterizing the different inhibitory behaviors of drugs toward kinase subfamilies, such as imatinib (aka Gleevec) toward $\mathrm{Abl} / \mathrm{Src} /$ Syk proteins. ${ }^{62,66,67}$ In this respect, it is interesting to note that resistance mechanisms involving the stabilization of the $\mathrm{KD}$ bridge might increase the association barrier for a neutral inhibitor such as PP1 without affecting the binding of the ATP natural substrate. This is due to the favorable interaction of the phosphate group of ATP with the Lys295, which does not require to break the $\mathrm{KD}$ bridge, as confirmed by $65 \%$ of the ATP-binding kinase structures that also present a formed $\mathrm{KD}$ bridge (see Figure S13). ${ }^{68}$

It is interesting to note that this mechanism was not identified by directly forcing the rupture of the $\mathrm{KD}$ salt bridge but by observing the effects of local hydration. This is consistent with statistical analysis performed on the Protein Data Bank, where it has been observed that one of the most relevant structural features of protein ligand binding is represented by water molecule architecture. ${ }^{69}$

In summary, binding processes involve several active components, including the solvent species, and unraveling their roles is an important task, made challenging by the temporal scale required to achieve the native binding and by the complex behavior of solvent-mediated effects. In this work, we mechanistically analyze the role of water in a biologically relevant and well-studied protein-ligand binding case: Src Kinase-PP1. Our analysis uses a novel computational approach belonging to the category of the so-called "enhanced sampling methods" in MD. These techniques increase the capabilities of $\mathrm{MD}$ by accelerating the simulation of transitions between configurations separated by high-energy barriers. We envision a campaign of several replicas of short ( $35 \mathrm{~ns}$ ) simulations where the conventional free dynamics of the solvated protein-ligand system is altered by a controlled repulsion between water and the heavy atoms found in the ligand and the binding site. Our simulations show that the behavior of water near the binding site surface during the biased simulations is similar to that which is known to occur in the proximity of hydrophobic sites.

Moreover, provided the applied bias is moderate, specific water interactions are preserved and can be discerned from those in which water acts merely as a bulk solvent. Focusing on the binding phenomenon, we compare our simulations with the longer and more computationally demanding plain $\mathrm{MD}$ simulations performed on the same system by the D. E. Shaw Research group, ${ }^{39}$ finding good qualitative agreement, including the presence of water-induced local energy minima encountered during the binding process. Interestingly, even though no specific knowledge about the reaction coordinate of the Src-PP1 association process was used in the bias, the correct binding conformation was observed in some of the replicas, as assessed by comparison with the crystal structure of the complex. Our analysis confirms that overcoming the desolvation penalty alone is not enough to guarantee the occurrence of a binding event, since there can be other relevant slow degrees of freedom. Application of the method to 
different systems may therefore perform differently and require some tuning of the parameters, such as which region of the protein is to be biased and which are suitable threshold values for the target hydration. Too-low target values would undesirably keep the bias alive after the encounter has occurred. The successful trajectories may be used as starting points for path-based free-energy calculation approaches. ${ }^{70,71}$

This approach allowed us to extend the study, leading to a striking observation from the biological and drug design points of view: we found that the entry of the ligand into the bindingsite cavity is concurrent with the breakage of a salt bridge in the site. This salt bridge, here named $\mathrm{KD}$, involves two residues, Lys295 and Asp404, which are remarkably evolutionarily preserved in the protein kinase family, acting like a molecular switch. We suggest that local hydration modulates this process of breaking and formation of the $\mathrm{KD}$ salt bridge, which in turn can modulate the binding efficiency toward the Src kinase along with the other Lys295-Glu310 salt bridge. Local hydration does in fact promote this breakage, and its fluctuations may allow conformational receptor-ligand adjustments that are typical of the induced-fit mechanism, facilitating in the end the binding of potential inhibitors. Src-PP1 is not the only system where the role of water molecules is central to defining the selectivity of a kinase inhibitor. ${ }^{72}$ Our structural bioinformatics analysis confirms that not just Lys295 and Asp404 but the polar interaction itself is very conserved in the kinase family and, especially, in the Tyr-kinase subfamily.

These observations lead to two pharmacological implications. First, as already conjectured elsewhere, ${ }^{73,74}$ localized water molecules, water networks, as well as the accessibility of solvent to the binding site, can be crucial factors to exploit when seeking for selectivity in the design of new ligands for members of promiscuous protein families. Second, the evolutionarily preserved $\mathrm{KD}$ salt bridge can act as a molecular switch in water-mediated binding, pointing to an Src conformational state that could be targeted in modern drug design.

Overall, we have shown that the localized tuning of hydration in an MD simulation can be a precious enabling tool to study relevant biological and biochemical processes. A delicate aspect of its application is the need to tune the strength of the bias on the analyzed system, in order to avoid an excess of force, which would hide subtler, but still relevant, aspects of the process. We are working to extend this kind of analysis to other fields such as material and nanosciences.

\section{ASSOCIATED CONTENT}

\section{s) Supporting Information}

The Supporting Information is available free of charge at https://pubs.acs.org/doi/10.1021/acs.jpclett.0c03075.

Methods: MD bias and protocol description. Identification of pocket and ligand placement. System setup for simulation RMSD and PP1-BS distance analysis. Illustrations of the following: Identification of residues in the binding site of Src kinase. Histogram of the number of water molecules at a given distance from the binding site of Src kinase. Standard deviation of the number of water molecules at a certain distance from the binding site of Src kinase. Distribution of the final PP1-Src distances. Probability distribution of the average minimum distance between PP1 and Src kinase. Number of $\mathrm{H}$ bonds on the binding site of Src, around PP1, and between Lys295-Asp404, Lys295-Glu310, and Lys295water along the trajectory unbiased of researchers at D. E. Shaw Research and in our biased trajectories. Distance between PP1 and the Src binding site and Lys295-Asp404 and Lys295-Glu310 along the PP1 entrance in the binding site. Comparative analysis of the distances between PP1 and the Src binding site and Lys295-Asp404 and Lys295-Glu310 along the PP1 entrance in the binding site for D. E. Shaw Research simulations and our biased trajectory. Structural conformation of Lys295, Glu310, and Asp404 in two ATP-Src crystal complexes with and without $\mathrm{Mg}^{2+}$ cation (PDF)

\section{Web-Enhanced Features}

Video of one of the successful binding replica simulations: PP1 is represented in orange licorice. K295, E310, G344, S345, and D404 residues are in licorice. Water molecules closer than $4 \AA$ from the SrcBS are in red-white CPK, while in green CPK are shown water molecules closer than $3 \AA$ from K295, E310, and $\mathrm{D} 404$. The protein is in iceblue cartoon. In blue dashed lines are the distances between K295[NZ]---[CG]D404 and K295[NZ]---[CD]E310. Finally, the protein residues closer than $3 \AA$ from the ligand are shown in lines. In the end of the movie, tested on the VLC player, the crystal pose of PP1 is added to the view.

\section{AUTHOR INFORMATION}

\section{Corresponding Authors}

Miguel A. Soler - CONCEPT Lab, Istituto Italiano di

Tecnologia, Genoa I-16163, Italy; 이 orcid.org/0000-00025780-9949; Email: miguel.soler@iit.it

Walter Rocchia - CONCEPT Lab, Istituto Italiano di Tecnologia, Genoa I-16163, Italy; 다이.org/0000-00032480-7151; Email: walter.rocchia@iit

\section{Authors}

Andrea Spitaleri - CONCEPT Lab, Istituto Italiano di Tecnologia, Genoa I-16163, Italy; Center for Omics Sciences, Emerging Bacterial Pathogens Unit, IRCCS San Raffaele Scientific Institute, Milan, Italy; ㅇo․ ord.org/0000-00033012-3557

Syeda R. Zia - CONCEPT Lab, Istituto Italiano di Tecnologia, Genoa I-16163, Italy; Dr. Panjwani Center for Molecular Medicine and Drug Research, International Center for Chemical and Biological Sciences, University of Karachi, Karachi 75270, Pakistan

Patrizio Di Micco - Cancer Research UK Cancer Therapeutics Unit, The Institute of Cancer Research, London SM2 5NG, U.K

Bissan Al-Lazikani - Cancer Research UK Cancer Therapeutics Unit, The Institute of Cancer Research, London SM2 5NG, U.K.

Complete contact information is available at: https://pubs.acs.org/10.1021/acs.jpclett.0c03075

\section{Author Contributions}

A.S., S.R.Z., and M.A.S. performed the computational simulations; M.A.S. and W.R. performed the analysis of the results; P.d.M. and B.A.L. performed the structural bioinformatics analysis; W.R. conceived and planned the computational experiments; M.A.S. and W.R. wrote the manuscript with the revisions of all authors. 


\section{Notes}

The authors declare the following competing financial interest(s): B.A.L. and P.d.M. are employees of the Institute of Cancer Research (ICR) which has a commercial interest in a range of drug targets, including protein kinases. The ICR operates a Rewards to Inventors scheme through which employees of the ICR may receive financial benefit following commercial licensing of a project. B.A.L. is currently or has been a consultant to Astex Pharmaceuticals, GSK, Astelas Pharma and Difiniens AG (member of Astra Zeneca group). B.A.L. is a former employee of Inpharmatica Ltd.W.R. and A.S. are co-founders of BiKi Technologies s.r.l., the company that sells the BiKi Life Sciences software suite.

\section{ACKNOWLEDGMENTS}

We thank D. E. Shaw Research for providing the unbiased trajectories of the Src-PP1 system. We acknowledge PRACE for awarding us access to Marconi at CINECA, Italy. We thank $\mathrm{K}$. Bulusu for his contributions to the early version of the protein kinase structure-led sequence alignment.

\section{REFERENCES}

(1) Levy, Y.; Onuchic, J. N. Water mediation in protein folding and molecular recognition. Annu. Rev. Biophys. Biomol. Struct. 2006, 35, $389-415$.

(2) Honig, B.; Nicholls, A. Classical electrostatics in biology and chemistry. Science 1995, 268 (5214), 1144-9.

(3) Sheinerman, F. B.; Norel, R.; Honig, B. Electrostatic aspects of protein-protein interactions. Curr. Opin. Struct. Biol. 2000, 10 (2), $153-9$.

(4) Garde, S. Physical chemistry: Hydrophobic interactions in context. Nature 2015, 517 (7534), 277-9.

(5) Chandler, D. Interfaces and the driving force of hydrophobic assembly. Nature 2005, 437 (7059), 640-7.

(6) Sali, A.; Shakhnovich, E.; Karplus, M. How does a protein fold? Nature 1994, 369 (6477), 248-51.

(7) Agashe, V. R.; Shastry, M. C.; Udgaonkar, J. B. Initial hydrophobic collapse in the folding of barstar. Nature 1995, 377 (6551), 754-7.

(8) Berne, B. J.; Weeks, J. D.; Zhou, R. Dewetting and hydrophobic interaction in physical and biological systems. Annu. Rev. Phys. Chem. 2009, 60, 85-103.

(9) Schneider, G.; Fechner, U. Computer-based de novo design of drug-like molecules. Nat. Rev. Drug Discovery 2005, 4 (8), 649-63.

(10) Schneider, P.; Walters, W. P.; Plowright, A. T.; Sieroka, N.; Listgarten, J.; Goodnow, R. A.; Fisher, J.; Jansen, J. M.; Duca, J. S.; Rush, T. S.; Zentgraf, M.; Hill, J. E.; Krutoholow, E.; Kohler, M.; Blaney, J.; Funatsu, K.; Luebkemann, C.; Schneider, G. Rethinking drug design in the artificial intelligence era. Nat. Rev. Drug Discovery 2020, 19, 353-364.

(11) Poornima, C. S.; Dean, P. M. Hydration in drug design. 3. Conserved water molecules at the ligand-binding sites of homologous proteins. J. Comput.-Aided Mol. Des. 1995, 9 (6), 521-31.

(12) Spyrakis, F.; Ahmed, M. H.; Bayden, A. S.; Cozzini, P.; Mozzarelli, A.; Kellogg, G. E. The Roles of Water in the Protein Matrix: A Largely Untapped Resource for Drug Discovery. J. Med. Chem. 2017, 60 (16), 6781-6827.

(13) Jukič, M.; Konc, J.; Gobec, S.; Janežič, D. Identification of Conserved Water Sites in Protein Structures for Drug Design. J. Chem. Inf. Model. 2017, 57 (12), 3094-3103.

(14) Khodadadi, S.; Sokolov, A. P. Atomistic details of protein dynamics and the role of hydration water. Biochim. Biophys. Acta, Gen. Subj. 2017, 1861, 3546-3552.

(15) Davis, A. M.; Teague, S. J.; Kleywegt, G. J. Application and limitations of X-ray crystallographic data in structure-based ligand and drug design. Angew. Chem., Int. Ed. 2003, 42 (24), 2718-36.
(16) Ebbinghaus, S.; Kim, S. J.; Heyden, M.; Yu, X.; Heugen, U.; Gruebele, M.; Leitner, D. M.; Havenith, M. An extended dynamical hydration shell around proteins. Proc. Natl. Acad. Sci. U. S. A. 2007, 104 (52), 20749-52.

(17) Dalvit, C.; Fogliatto, G.; Stewart, A.; Veronesi, M.; Stockman, B. WaterLOGSY as a method for primary NMR screening: practical aspects and range of applicability. J. Biomol. NMR 2001, 21 (4), 34959.

(18) Dopfer, O.; Fujii, M. Probing Solvation Dynamics around Aromatic and Biological Molecules at the Single-Molecular Level. Chem. Rev. 2016, 116 (9), 5432-63.

(19) Conti Nibali, V.; Havenith, M. New insights into the role of water in biological function: studying solvated biomolecules using terahertz absorption spectroscopy in conjunction with molecular dynamics simulations. J. Am. Chem. Soc. 2014, 136 (37), 12800-7.

(20) Bortolato, A.; Deflorian, F.; Weiss, D. R.; Mason, J. S. Decoding the Role of Water Dynamics in Ligand-Protein Unbinding: CRF1R as a Test Case. J. Chem. Inf. Model. 2015, 55 (9), 1857-66.

(21) Bortolato, A.; Tehan, B. G.; Smith, R. T.; Mason, J. S. Methodologies for the Examination of Water in GPCRs. Methods Mol. Biol. 2018, 1705, 207-232.

(22) Bodnarchuk, M. S. Water, water, everywhere $\cdots$ It's time to stop and think. Drug Discovery Today 2016, 21 (7), 1139-46.

(23) Sindhikara, D. J.; Hirata, F. Analysis of biomolecular solvation sites by 3D-RISM theory. J. Phys. Chem. B 2013, 117 (22), 6718-23.

(24) Setny, P.; Baron, R.; Michael Kekenes-Huskey, P.; McCammon, J. A.; Dzubiella, J. Solvent fluctuations in hydrophobic cavity-ligand binding kinetics. Proc. Natl. Acad. Sci. U. S. A. 2013, 110 (4), 1197-202.

(25) Weiß, R. G.; Setny, P.; Dzubiella, J. Principles for Tuning Hydrophobic Ligand-Receptor Binding Kinetics. J. Chem. Theory Comput. 2017, 13 (6), 3012-3019.

(26) Balius, T. E.; Fischer, M.; Stein, R. M.; Adler, T. B.; Nguyen, C. N.; Cruz, A.; Gilson, M. K.; Kurtzman, T.; Shoichet, B. K. Testing inhomogeneous solvation theory in structure-based ligand discovery. Proc. Natl. Acad. Sci. U. S. A. 2017, 114 (33), E6839-E6846.

(27) Ball, P. Biophysics: More than a bystander. Nature 2011, 478 (7370), 467-8.

(28) Patel, A. J.; Varilly, P.; Jamadagni, S. N.; Hagan, M. F.; Chandler, D.; Garde, S. Sitting at the edge: how biomolecules use hydrophobicity to tune their interactions and function. J. Phys. Chem. B 2012, 116 (8), 2498-503.

(29) Hummer, G. Molecular binding: Under water's influence. Nat. Chem. 2010, 2 (11), 906-7.

(30) Graziano, G. Molecular driving forces of the pocket-ligand hydrophobic association. Chem. Phys. Lett. 2012, 533, 95-99.

(31) Setny, P.; Baron, R.; McCammon, J. A. How Can Hydrophobic Association Be Enthalpy Driven? J. Chem. Theory Comput. 2010, 6 (9), 2866-2871.

(32) Kinoshita, M.; Hayashi, T. Unified elucidation of the entropydriven and -opposed hydrophobic effects. Phys. Chem. Chem. Phys. 2017, 19 (38), 25891-25904.

(33) Baron, R.; Setny, P.; McCammon, J. A. Water in cavity-ligand recognition. J. Am. Chem. Soc. 2010, 132 (34), 12091-7.

(34) Grossman, M.; Born, B.; Heyden, M.; Tworowski, D.; Fields, G. B.; Sagi, I.; Havenith, M. Correlated structural kinetics and retarded solvent dynamics at the metalloprotease active site. Nat. Struct. Mol. Biol. 2011, 18 (10), 1102-8.

(35) Ross, G. A.; Bodnarchuk, M. S.; Essex, J. W. Water Sites, Networks, And Free Energies with Grand Canonical Monte Carlo. J. Am. Chem. Soc. 2015, 137 (47), 14930-43.

(36) Pan, A. C.; Borhani, D. W.; Dror, R. O.; Shaw, D. E. Molecular determinants of drug-receptor binding kinetics. Drug Discovery Today 2013, 18 (13-14), 667-73.

(37) Ladbury, J. E. Just add water! The effect of water on the specificity of protein-ligand binding sites and its potential application to drug design. Chem. Biol. 1996, 3 (12), 973-80.

(38) Young, T.; Abel, R.; Kim, B.; Berne, B. J.; Friesner, R. A. Motifs for molecular recognition exploiting hydrophobic enclosure in 
protein-ligand binding. Proc. Natl. Acad. Sci. U. S. A. 2007, 104 (3), 808-13.

(39) Shan, Y.; Kim, E. T.; Eastwood, M. P.; Dror, R. O.; Seeliger, M. A.; Shaw, D. E. How does a drug molecule find its target binding site? J. Am. Chem. Soc. 2011, 133 (24), 9181-3.

(40) Jukič, M.; Konc, J.; Janežič, D.; Bren, U. ProBiS H2O MD Approach for Identification of Conserved Water Sites in Protein Structures for Drug Design. ACS Med. Chem. Lett. 2020, 11 (5), 877882.

(41) Decherchi, S.; Berteotti, A.; Bottegoni, G.; Rocchia, W.; Cavalli, A. The ligand binding mechanism to purine nucleoside phosphorylase elucidated via molecular dynamics and machine learning. Nat. Commun. 2015, 6, 6155.

(42) Dror, R. O.; Pan, A. C.; Arlow, D. H.; Borhani, D. W.; Maragakis, P.; Shan, Y.; Xu, H.; Shaw, D. E. Pathway and mechanism of drug binding to G-protein-coupled receptors. Proc. Natl. Acad. Sci. U. S. A. 2011, 108 (32), 13118-23.

(43) Buch, I.; Giorgino, T.; De Fabritiis, G. Complete reconstruction of an enzyme-inhibitor binding process by molecular dynamics simulations. Proc. Natl. Acad. Sci. U. S. A. 2011, 108 (25), 10184-9.

(44) Beuming, T.; Che, Y.; Abel, R.; Kim, B.; Shanmugasundaram, V.; Sherman, W. Thermodynamic analysis of water molecules at the surface of proteins and applications to binding site prediction and characterization. Proteins: Struct., Funct., Genet. 2012, 80 (3), 871-83.

(45) Robinson, D. D.; Sherman, W.; Farid, R. Understanding kinase selectivity through energetic analysis of binding site waters. ChemMedChem 2010, 5 (4), 618-27.

(46) Barillari, C.; Duncan, A. L.; Westwood, I. M.; Blagg, J.; van Montfort, R. L. Analysis of water patterns in protein kinase binding sites. Proteins: Struct., Funct., Genet. 2011, 79 (7), 2109-21.

(47) Stank, A.; Kokh, D. B.; Fuller, J. C.; Wade, R. C. Protein Binding Pocket Dynamics. Acc. Chem. Res. 2016, 49 (5), 809-15.

(48) Spitaleri, A.; Decherchi, S.; Cavalli, A.; Rocchia, W. Fast Dynamic Docking Guided by Adaptive Electrostatic Bias: The MDBinding Approach. J. Chem. Theory Comput. 2018, 14 (3), 17271736.

(49) Dixit, A.; Verkhivker, G. M. Structure-functional prediction and analysis of cancer mutation effects in protein kinases. Comput. Math. Methods Med. 2014, 2014, 653487.

(50) Wu, P.; Nielsen, T. E.; Clausen, M. H. FDA-approved smallmolecule kinase inhibitors. Trends Pharmacol. Sci. 2015, 36 (7), 42239.

(51) Foda, Z. H.; Shan, Y.; Kim, E. T.; Shaw, D. E.; Seeliger, M. A. A dynamically coupled allosteric network underlies binding cooperativity in Src kinase. Nat. Commun. 2015, 6, 5939.

(52) Vlahovic, G.; Crawford, J. Activation of tyrosine kinases in cancer. Oncologist 2003, 8 (6), 531-8.

(53) Zia, S. R.; Gaspari, R.; Decherchi, S.; Rocchia, W. Probing Hydration Patterns in Class-A GPCRs via Biased MD: The A2A Receptor. J. Chem. Theory Comput. 2016, 12 (12), 6049-6061.

(54) Weiß, R. G.; Setny, P.; Dzubiella, J. Solvent Fluctuations Induce Non-Markovian Kinetics in Hydrophobic Pocket-Ligand Binding. J. Phys. Chem. B 2016, 120 (33), 8127-36.

(55) Roskoski, R. A historical overview of protein kinases and their targeted small molecule inhibitors. Pharmacol. Res. 2015, 100, 1-23.

(56) Meharena, H. S.; Fan, X.; Ahuja, L. G.; Keshwani, M. M.; McClendon, C. L.; Chen, A. M.; Adams, J. A.; Taylor, S. S. Decoding the Interactions Regulating the Active State Mechanics of Eukaryotic Protein Kinases. PLoS Biol. 2016, 14 (11), No. e2000127.

(57) McSkimming, D. I.; Rasheed, K.; Kannan, N. Classifying kinase conformations using a machine learning approach. BMC Bioinf. 2017, 18 (1), 86.

(58) Modi, V.; Dunbrack, R. L. Defining a new nomenclature for the structures of active and inactive kinases. Proc. Natl. Acad. Sci. U. S. A. 2019, 116 (14), 6818-6827.

(59) Lin, Y. L.; Roux, B. Computational analysis of the binding specificity of Gleevec to Abl, c-Kit, Lck, and c-Src tyrosine kinases. J. Am. Chem. Soc. 2013, 135 (39), 14741-53.
(60) Decherchi, S.; Spitaleri, A.; Stone, J.; Rocchia, W. NanoShaperVMD interface: computing and visualizing surfaces, pockets and channels in molecular systems. Bioinformatics 2019, 35 (7), 12411243.

(61) Bulusu, K. C.; Tym, J. E.; Coker, E. A.; Schierz, A. C.; AlLazikani, B. canSAR: updated cancer research and drug discovery knowledgebase. Nucleic Acids Res. 2014, 42 (D1), D1040-7.

(62) Jiao, Q.; Bi, L.; Ren, Y.; Song, S.; Wang, Q.; Wang, Y. S. Advances in studies of tyrosine kinase inhibitors and their acquired resistance. Mol. Cancer 2018, 17 (1), 36.

(63) Shan, Y.; Seeliger, M. A.; Eastwood, M. P.; Frank, F.; Xu, H.; Jensen, M.; Dror, R. O.; Kuriyan, J.; Shaw, D. E. A conserved protonation-dependent switch controls drug binding in the $\mathrm{Abl}$ kinase. Proc. Natl. Acad. Sci. U. S. A. 2009, 106 (1), 139-44.

(64) Hari, S. B.; Merritt, E. A.; Maly, D. J. Sequence determinants of a specific inactive protein kinase conformation. Chem. Biol. 2013, 20 (6), 806-15.

(65) Persky, N. S.; Hernandez, D.; Do Carmo, M.; Brenan, L.; Cohen, O.; Kitajima, S.; Nayar, U.; Walker, A.; Pantel, S.; Lee, Y.; Cordova, J.; Sathappa, M.; Zhu, C.; Hayes, T. K.; Ram, P.; Pancholi, P.; Mikkelsen, T. S.; Barbie, D. A.; Yang, X.; Haq, R.; Piccioni, F.; Root, D. E.; Johannessen, C. M. Defining the landscape of ATPcompetitive inhibitor resistance residues in protein kinases. Nat. Struct. Mol. Biol. 2020, 27 (1), 92-104.

(66) Meng, Y.; Pond, M. P.; Roux, B. Tyrosine Kinase Activation and Conformational Flexibility: Lessons from Src-Family Tyrosine Kinases. Acc. Chem. Res. 2017, 50 (5), 1193-1201.

(67) Agafonov, R. V.; Wilson, C.; Otten, R.; Buosi, V.; Kern, D. Energetic dissection of Gleevec's selectivity toward human tyrosine kinases. Nat. Struct. Mol. Biol. 2014, 21 (10), 848-53.

(68) Mondal, J.; Tiwary, P.; Berne, B. J. How a Kinase Inhibitor Withstands Gatekeeper Residue Mutations. J. Am. Chem. Soc. 2016, 138 (13), 4608-15.

(69) Boström, J.; Hogner, A.; Schmitt, S. Do structurally similar ligands bind in a similar fashion? J. Med. Chem. 2006, 49 (23), 671625 .

(70) Branduardi, D.; Gervasio, F. L.; Parrinello, M. From A to B in free energy space. J. Chem. Phys. 2007, 126 (5), No. 054103.

(71) Maragliano, L.; Fischer, A.; Vanden-Eijnden, E.; Ciccotti, G. String method in collective variables: minimum free energy paths and isocommittor surfaces. J. Chem. Phys. 2006, 125 (2), 24106.

(72) Levinson, N. M.; Boxer, S. G. A conserved water-mediated hydrogen bond network defines bosutinib's kinase selectivity. Nat. Chem. Biol. 2014, 10 (2), 127-32.

(73) Re, S.; Oshima, H.; Kasahara, K.; Kamiya, M.; Sugita, Y. Encounter complexes and hidden poses of kinase-inhibitor binding on the free-energy landscape. Proc. Natl. Acad. Sci. U. S. A. 2019, 116 (37), 18404-18409.

(74) Krimmer, S. G.; Cramer, J.; Betz, M.; Fridh, V.; Karlsson, R.; Heine, A.; Klebe, G. Rational Design of Thermodynamic and Kinetic Binding Profiles by Optimizing Surface Water Networks Coating Protein-Bound Ligands. J. Med. Chem. 2016, 59 (23), 10530-10548. 